SLAC-PUB-14644

LCLS-TN-11-4

October 19, 2011

\title{
What have we learned from the LCLS injector?*
}

Feng Zhou and Axel Brachmann for the LCLS injector team

The LCLS injector reliably delivered a high quality electron beam since it started operations three years ago. Some initial commissioning results were described in Ref. [1]. This note is to summarize what we have understood from the LCLS injector including drive laser, cathode, RF gun, injector beam line elements, beam modeling and operations.

\section{LCLS drive laser system}

What we have learned during the LCLS injector commissioning and operation has lead to modified specifications for the UV drive laser system. A flat temporal profile originally required in the LCLS design is not necessary to achieve a low emittance electron beam for the LCLS operations. According to our recent studies, a laser pulse with 3 ps Gaussian temporal profile (FWHM) can achieve a similar emittance [2] compared to a flat temporal profile laser. The sensitivity of the emittance to the laser pulse length at $250 \mathrm{pC}$ of bunch charge was studied, as shown in Figure 1. Only slight variations were observed for both projected and time-sliced emittances with laser pulses from 2.2 ps to 4.5 ps FWHM. Space charge forces certainly become weak but RF emittance which is proportional to the bunch length square increases due to the longer laser pulse. Thus, the projected emittance optimum may be a trade-off between space charge forces and RF emittance, while the time-sliced emittance determined by space charge forces may be slightly improved with a longer laser pulse length. Simulations and theory [3] also show that spatial Gaussian-cut laser profile produces a better emittance compared to uniform profiles due to increased linearity of the space charge forces. Preliminary experimental data [3] show that the emittance with a Gaussian-cut is almost as good as the one with uniform although not better than. In addition, the laser transmission through the iris for spatial Gaussian-cut is about twice the one for spatial uniform. More experimental studies with the new Coherent laser system are needed to make a more solid conclusion.

$100 \mu \mathrm{J}$ of laser is nominally required for operations of $250 \mathrm{pC}$ charge assume QE is $\sim 4 \times 10^{-5}$. It is suggested to have more head room for laser energy in the LCLS-II for a potential QE degradation of the cathode. Note that some optical damage issues had been observed [4] in the LCLS drive laser system when the laser energy exceeded $100 \mu \mathrm{J}$. When the laser energy and the cathode's QE are sufficient to provide tuning overhead, we plan to study the reduction of the thermal emittance by tuning the laser to a longer wave length [5]. Therefore the LCLS-II laser is required to be tunable within $10-20 \mathrm{~nm}$ to reserve this possibility.

Currently, the LCLS injector uses the Thales drive laser, which has a large bandwidth and more pulse stretching is needed to reach 3-4 ps after pulse compression. Furthermore, the spatial profile is not so

\footnotetext{
* Work supported in part by the DOE Contract DE-AC02-76SF00515. This work was performed in support of the LCLS project at SLAC.
} 
smooth. The LCLC-I and LCLS-II injectors plan to adopt the Coherent laser system, which may overcome the drawbacks of the Thales laser.
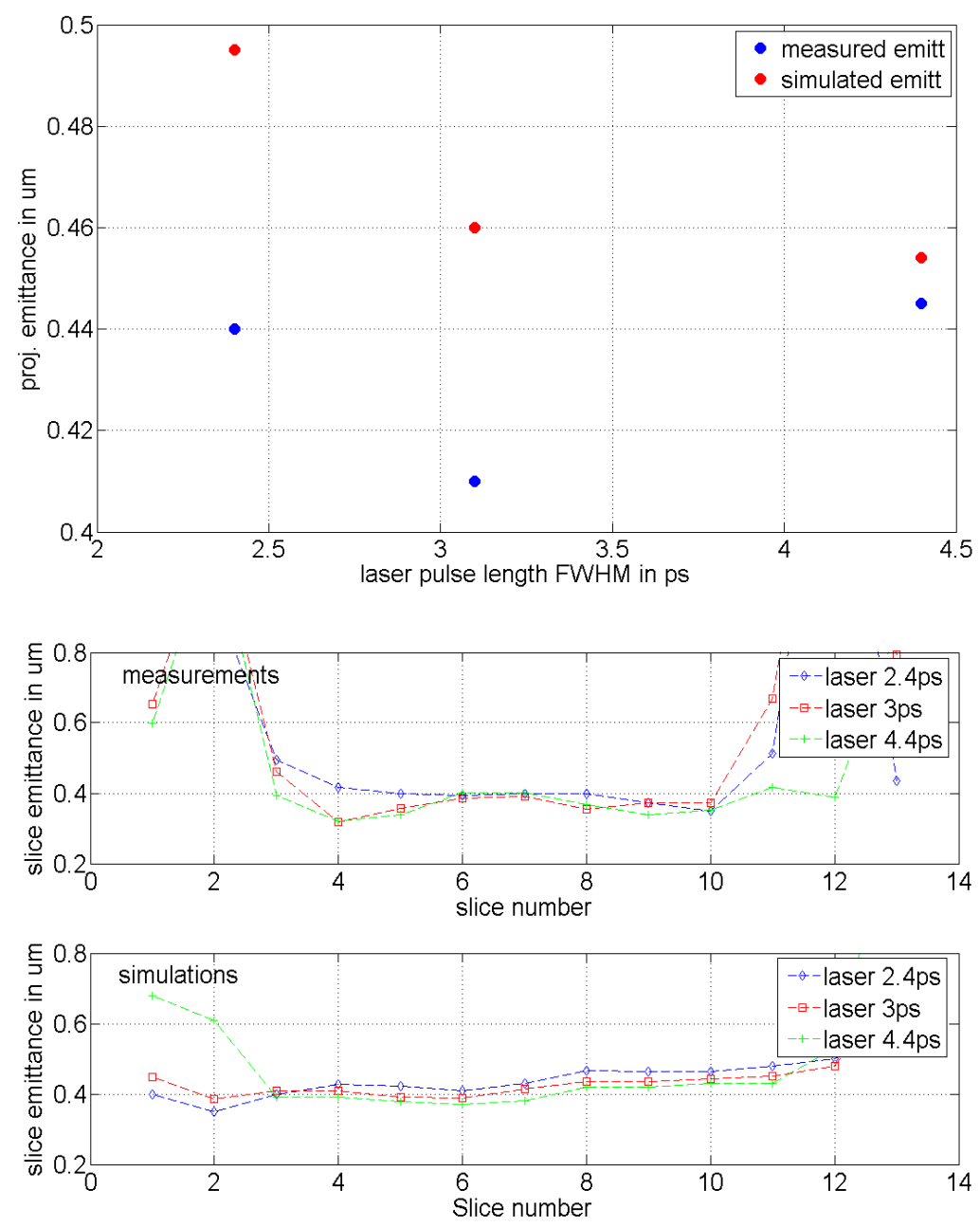

Figure 1: Measured and simulated emittances (projected and time-sliced) vs. laser pulse length at $250 \mathrm{pC}$ of bunch charge.

\section{LCLS Cathode \& RF gun}

The LCLS injector has used three copper cathodes to date. The first cathode had a low QE but was sufficient for initial commissioning. The second cathode lasted for almost three years of user operations from July 2008 to May 2011. Its QE was $4 \times 10^{-5}$ [6] but quickly decayed to half within 7-10 days when the LCLS operated at $120 \mathrm{~Hz}$. For this reason the laser location had to be moved frequently to find new high QE areas on the cathode. This movement and subsequent retuning of the injector occupied significant machine time and only a limited number of locations can deliver the desired low emittance electron beam. The third cathode started operations in May 2011, and its original QE was $\sim 5 \times 10^{-6}$, which was insufficient for users operations. On July 4, 2011 we performed laser cleaning, which increased the QE to $\sim 5 \times 10^{-5}$. Over time, the QE continues to increase from $5 \times 10^{-5}$ to currently $1 \times 10^{-4}$ during regular 
operations. The gun vacuum has gradually improved and is still decreasing shown in Figure 2 . The mechanism of the QE increase during the operations is not fully understood yet but it is probably correlated to gun vacuum improvement. The emittance evolution after laser cleaning is shown in Figure 3. It is shown that it takes 1-2 weeks to achieve an ultra-small emittance electron beam after laser cleaning. In addition, both the emittance and QE of a cleaned spot can be improved even when it is idle (i.e., no laser operates on the spot) over time. On July 26, 2011 a spot was cleaned using laser and its immediately measured $\mathrm{QE}$ and emittance was $5 \times 10^{-5}$ and $0.74 \mu \mathrm{m}\left(\varepsilon_{\mathrm{x}}\right) / 0.55 \mu \mathrm{m}\left(\varepsilon_{\mathrm{y}}\right)$ (for $150 \mathrm{pC}$ ), respectively. Then, the laser was moved to another spot rather than the newly cleaned spot on the cathode for regular operations. Six weeks later, improvement of QE and emittance of the newly cleaned spot was observed: QE and projected emittance become $6 \times 10^{-5}$ and $0.54 \mu \mathrm{m}\left(\varepsilon_{\mathrm{x}}\right) / 0.48 \mu \mathrm{m}\left(\varepsilon_{\mathrm{y}}\right)$ (for $250 \mathrm{pC}$ ) respectively. The mechanism of the emittance improvement is not fully understood yet but it is probably due to more uniform surface smoothened by RF conditioning and or regular laser operation.

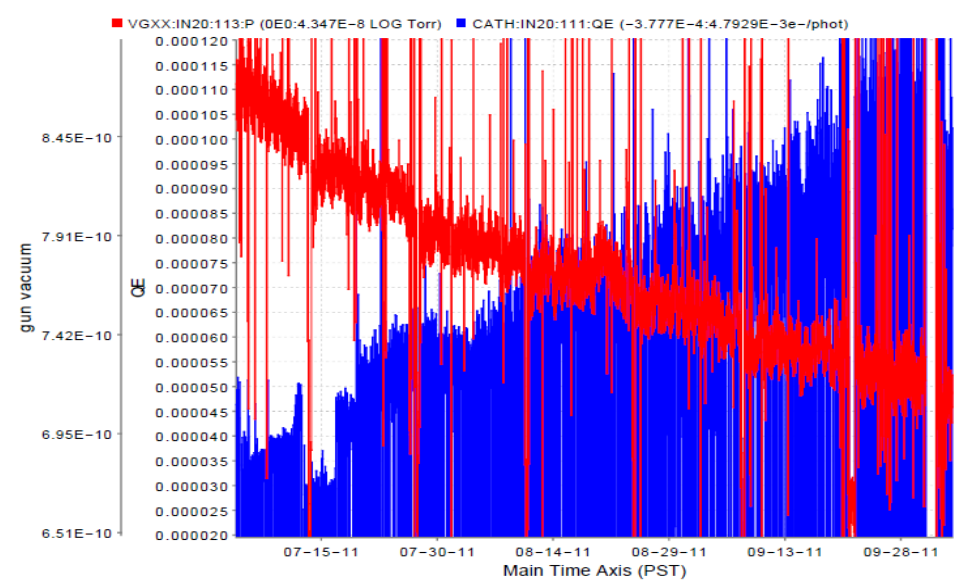

Figure 2: QE (blue) and gun vacuum (red) evolutions from July 4 to October 4, 2011. Note that the spot used for operations was cleaned with laser on July 4, 2011

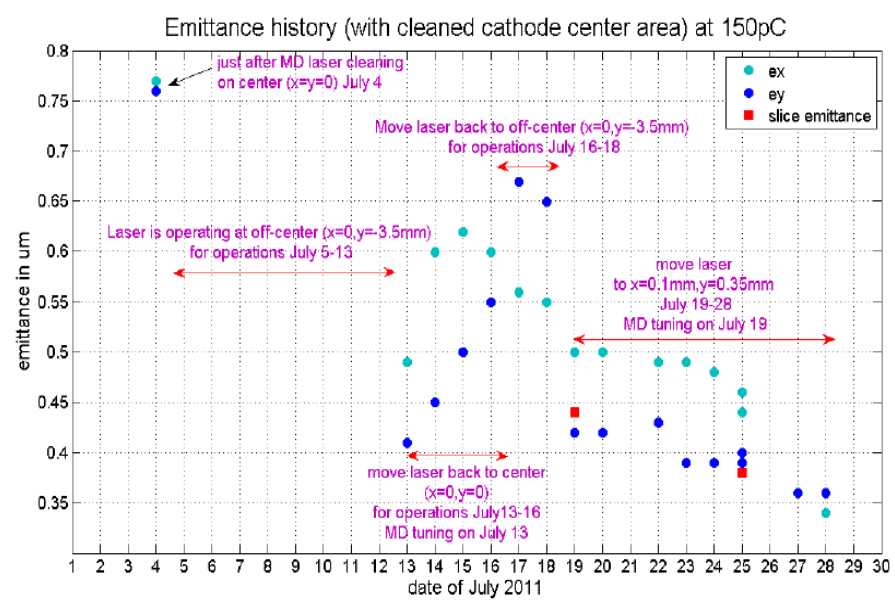

Figure 3: Emittance evolution from July 4 to July 28, 2011 (150 pC of bunch charge). Note that the spot used for operations was cleaned with laser on July 4, 2011. 
The originally required tolerance of drive laser location on the cathode with respect to gun center was stringent, only $100 \mu \mathrm{m}$. However, the LCLS commissioning and operational experience have shown a range, $\pm 2 \mathrm{~mm}$ of the laser location on the cathode with respect to solenoid center is sufficient to produce desired low emittance electron beam. Figure 4 shows the projected and time-sliced emittances with $250 \mathrm{pC}$ of bunch charge. It illustrates a small increase of the projected emittance, while the timesliced emittance remains almost unchanged with a $2.2 \mathrm{~mm}$ offset in comparison with on-center beam performance. An offset beam experiences a time-dependent RF kick, which impacts the projected emittance but has a smaller effect on the time-sliced emittance. Figure 5 compares the thermal emittance of the second and third cathodes. An extensive dataset is available for the second cathode (no cleaning treatments in the gun other except the normal procedures during manufacturing and installation). As described above, the third cathode was laser cleaned. It is shown that after a few months of operations the thermal emittance of the laser-cleaned cathode recovers to the level of the second cathode.
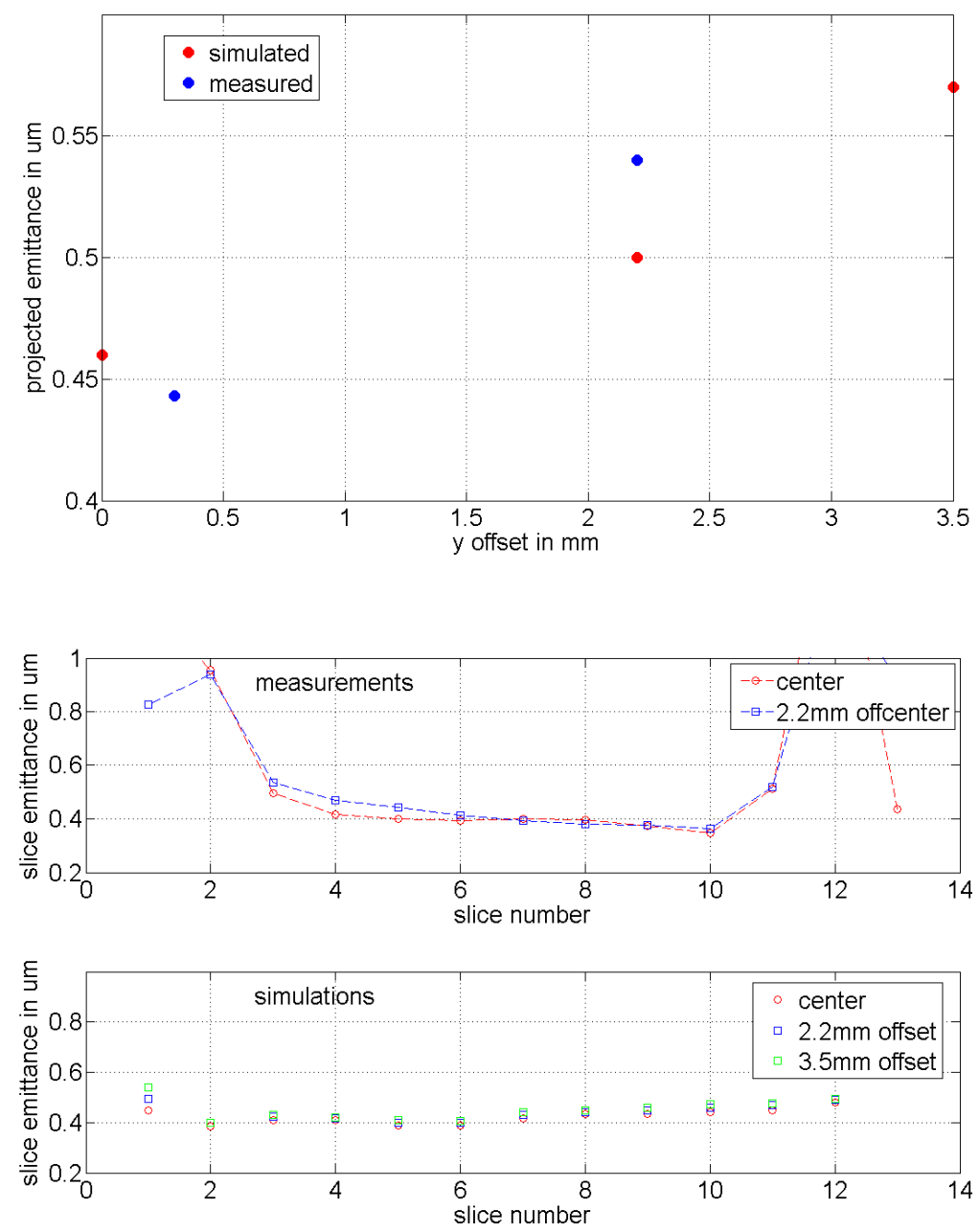

Figure 4: Projected and time-sliced emittances with beam offset on cathode ( $250 \mathrm{pC}$ ). 


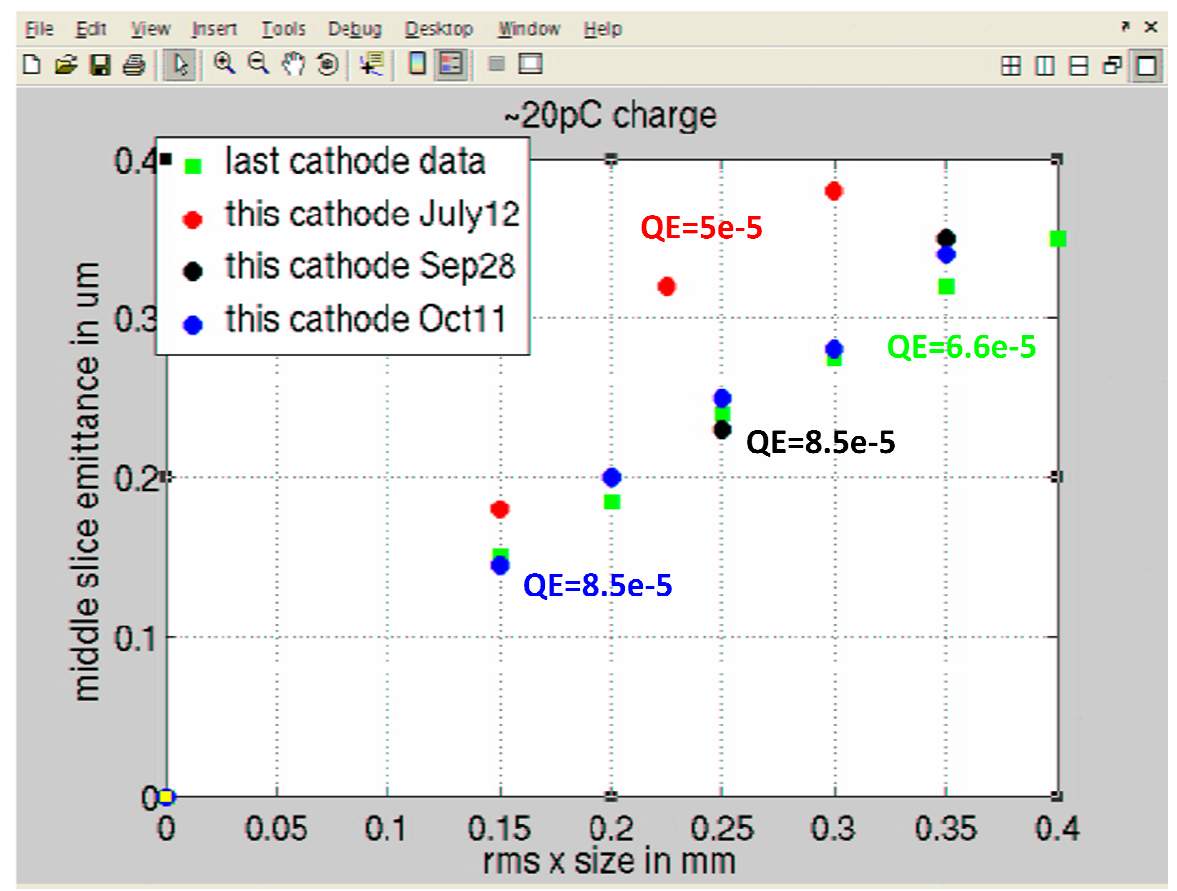

Figure 5: Thermal emittance measurements for the last and current cathodes.

The LCLS gun was fabricated based on an improved BNL/SLAC/UCLA gun-III design. A number of notable improvements [7] have been incorporated, for example, increasing RF modes separation to minimize RF mode beating, cancelling dipole and quadrupoles in the gun and linac coupler cells, and integrating an emittance compensation solenoid field with mini quadrupole correctors. The LCLS-II gun will duplicate the LCLS-I gun except the replacement of dual-RF windows to single-RF window [8].

Injector beam line elements and diagnostics

A few correctors and the second solenoid located immediately upstream of LOA and some beam diagnostics had been hardly used in the LCLS injector commissioning and operations. They will be deleted in the LCLS-II injector beam line. Some elements, for example, vacuum valve, ion pumps, RGA, two Faraday cups are added to the LCLS-II beam line. The detailed element list of the deletions and additions to the LCLS-II injector beam line with respect to LCLS-I injector is described in Ref. [9].

\section{Injector modeling \& operational issues}

The LCLS injector modeling using ImpactT code agrees with measurements. The difference between the measurements and simulations is within $10-20 \%$. We are also able to apply a realistic spatial laser beam as input distributions for particle tracking.

The LCLS injector (laser systems included) operates with a high availability ( 97\%) [10]. Downtime are mostly related to the LLRF systems, for example, lost RF synchronization of the mode locked seed laser, laser/gun/LOA/LOB RF amplitude and phase jittering and unexpected klystron cycling. Our laser 
and RF experts are addressing these issues with goal to improve future availability of the LCLS-I and II injectors.

\section{Acknowledgements}

We would like to thank the LCLS dedicated team for injector commissioning and operations, particularly David Dowell, Paul Emma, and the dedicated laser group.

\section{References}

1. LCLS commissioning team, PRST-AB 11, 030703 (2008).

2. P. Emma, et al., LCLS E-log, 2010.

3. F. Zhou, et al., FEL 2011.

4. W. White, private communication, 2011.

5. C. Hauri, et al., PRL 104, 234802 (2010).

6. A. Brachmann, et al., IPAC11, 2011.

7. D. Dowell, FLS2010, 2010.

8. E. Jongewaard, private communication, 2011.

9. P. Emma, list of changes for LCLS-II injector with respect to LCLS-I, 2011.

10. W. Colocho, private communication, 2011. 